\title{
Solution of multi-player linear-quadratic
}

\section{alternating-move games and its application to the} timing pattern of wage adjustment

\author{
Sau-Him Paul Lau* \\ University of Hong Kong \\ This version: March 2001
}

\begin{abstract}
In order to analyze a multi-player linear-quadratic alternating-move dynamic game, this paper develops a solution method by making use of the similar structure of this game and a simultaneous-move dynamic game with alternating payoff functions. It then applies the method to investigate whether staggered or synchronized wage adjustment will be preferred when more than two wage setters interact strategically and dynamically. The results suggest that the strategic benefit provided by staggered wage adjustment is robust with respect to the number of sectors in the economy.
\end{abstract}

JEL Classification Numbers: C73; E24

Key words: alternating moves; multi-player; pattern of wage adjustment

*Correspondence: S.-H. P. Lau, School of Economics and Finance, University of Hong Kong, Pokfulam Road, Hong Kong. E-mail: laushp@hkucc.hku.hk. I am grateful to Gaby Lozada, HonYuen Tam, John Taylor and a referee for helpful comments and discussions. 


\section{INTRODUCTION}

Linear-quadratic alternating-move dynamic games with two players have been used in Cyert and DeGroot (1970), Maskin and Tirole (1987), De Fraja (1993), Tanaka (1994), Lau (1996) and Cahuc and Kempf (1997). In these games, each player's choice variable lasts for two periods, and the players' action times are alternating such that one player sets his control at every even period, while his opponent chooses at every odd period.

As some economic issues (such as the aggregate timing pattern of wage or price adjustment in a multi-sector model) may involve the analysis of strategic and dynamic interaction among many players, this paper develops a general solution method to the class of linear-quadratic alternating-move games with $n(n \geq 2)$ players. Each player's action lasts for $n$ periods, and they choose their controls alternatingly. Extending the method for simultaneous-move dynamic games considered in Kydland (1975), this paper presents an algorithm to solve linear-quadratic alternating-move games. Moreover, verifiable conditions which guarantee the existence and uniqueness of the solution are given.

Another motivation of this paper is to investigate whether a staggered timing pattern will be preferred when more than two wage setters interact strategically and dynamically. Lau (1996) studies two aspects of labor market institutions (staggered versus synchronized wage setting, and coordinated versus noncooperative wage adjustment) in an intertemporal model with two players. In particular, it is shown that the two wage setters prefer moving alternatingly when they interact noncooperatively. By moving alternatingly, the wage setters are able to commit temporarily not to offset the effects of each other's action. The strategic benefit of staggered wage adjustment provides a justification for the assumption of staggered timing pattern used in Fischer (1977) and Taylor (1980). 
In previous research on the microfoundation of staggered moves, Fethke and Policano (1986) show that when the number of sectors increases in their earlier model (Fethke and Policano, 1984) which emphasizes the transmission of employment effects across sectors, it becomes less likely that staggered wage adjustment will be optimal. This is because "... the effect on the aggregate price level of the actions by any one sector is negligible, and the beneficial externality associated with staggered negotiation is too small to have an effect." (Fethke and Policano, 1986, p. 872). In light of their results, it is interesting to examine whether the strategic benefit provided by staggered wage adjustment is robust with respect to the number of wage setters in the model economy.

The remaining parts of this paper proceed as follows. Section 2 considers a twosymmetric-player alternating-move game, and shows how it can be reformulated as a simultaneous-move dynamic game with alternating payoff functions. Generalizing from this idea, Section 3 then presents a solution method to obtain the equilibrium decision rules of multi-player linear-quadratic alternating-move games. Section 4 studies the question of synchronized versus staggered wage adjustment in an economy with more than two sectors. Section 5 provides conclusions.

\section{A MOTIVATING EXAMPLE}

Maskin and Tirole (1987) provide elegant analysis of an alternating-move game with two symmetric players and apply it to a dynamic quantity (or capacity) competition model. A similar formulation has been followed and used in other papers such as De Fraja (1993) and Cahuc and Kempf (1997). This section considers a two-symmetricplayer linear-quadratic alternating-move game with general deterministic quadratic objective functions, which includes the various slightly different functional forms used by Maskin and Tirole (1987), De Fraja (1993) and Cahuc and Kempf (1997) as special cases. It shows how this game can be reformulated as a simultaneous-move 
dynamic game with alternating payoff functions. The analysis of this section helps clarify the underlying idea behind the reformulation procedure. This reformulation procedure, which is easier to understand in a two-player model, will be generalized to a multi-player model in the Section 3. The reformulation is an important step for the algorithm suggested in this paper.

The alternating-move game discussed in this section consists of two symmetric players, labelled as players 0 and 1. Player 0 (resp. 1) chooses a strategic variable $z$ at every even (resp. odd) period, which lasts for two periods. As a result, $z_{0,2 \lambda+1}=z_{0,2 \lambda}$ and $z_{1,2 \lambda}=z_{1,2 \lambda-1}$, where $z_{i, t}$ denotes the choice of player $i$ at period $t$, which is set either in the current or previous period. Each player maximizes an intertemporal payoff function, which is given by

$$
\sum_{t=0}^{r-1} \beta^{t} U_{i, t},
$$

where $0<\beta<1$, and the single-period payoff function $U_{i, t}$ is given by:

$$
U_{i, t}=a_{0}\left(z_{i, t}\right)^{2}+a_{1}\left(z_{j, t}\right)^{2}+2 a_{2} z_{i, t} z_{j, t}+2 a_{3} z_{i, t}+2 a_{4} z_{j, t}+a_{5},
$$

with $i, j=0,1$ and $i \neq j$, and $a_{0}<0$.

In order to apply the solution method suggested in this paper, it is helpful to reformulate the alternating-move game as a simultaneous-move dynamic game with alternating payoff functions. For the above game, the first step of the reformulation procedure is to define $x_{0,2 \lambda}=z_{0,2 \lambda}$ and $x_{1,2 \lambda+1}=z_{1,2 \lambda+1}$. Note that $z_{0,2 \lambda}$ (resp. $z_{1,2 \lambda+1}$ ) is the choice of player 0 (resp. player 1 ) when he moves. The second step is to introduce dummy control variables $x_{1,2 \lambda}\left(\right.$ resp. $\left.x_{0,2 \lambda+1}\right)$ for player 1 (resp. player 0 ) when his opponent moves. The third step is to define the vector of controls $x_{t} \equiv$ $\left(x_{0, t}, x_{1, t}\right)^{\prime}$ for the new game, and the vector of state variables

$$
y_{t} \equiv\left(x_{0, t-1}, x_{1, t-1}, x_{0, t-2}, x_{1, t-2}, 1\right)^{\prime}
$$


As a result, the law of motion for the simultaneous-move game is represented by:

$$
y_{t+1}=A y_{t}+B x_{t}=\left[\begin{array}{ccccc}
0 & 0 & 0 & 0 & 0 \\
0 & 0 & 0 & 0 & 0 \\
1 & 0 & 0 & 0 & 0 \\
0 & 1 & 0 & 0 & 0 \\
0 & 0 & 0 & 0 & 1
\end{array}\right] y_{t}+\left[\begin{array}{ll}
1 & 0 \\
0 & 1 \\
0 & 0 \\
0 & 0 \\
0 & 0
\end{array}\right] x_{t} \text {. }
$$

The last step is to express the players' payoff functions in terms of the variables of the reformulated simultaneous-move game, instead of the variables of the original game. It can be observed that, except for the inclusion of an extra term $\delta\left(x_{i, t}\right)^{2}$ in (6), the single-period payoff function (2) for the original game is equivalent to:

$$
\begin{gathered}
U_{i, t}=a_{0}\left(x_{i, t}\right)^{2}+a_{1}\left(x_{j, t-1}\right)^{2}+2 a_{2} x_{i, t} x_{j, t-1}+2 a_{3} x_{i, t}+2 a_{4} x_{j, t-1}+a_{5} \\
\equiv y_{t+1}^{\prime} Q_{i, k} y_{t+1}
\end{gathered}
$$

for $i=0$ (if $t$ is even) or $i=1$ (if $t$ is odd), and

$$
\begin{gathered}
U_{i, t}=\delta\left(x_{i, t}\right)^{2}+a_{0}\left(x_{i, t-1}\right)^{2}+a_{1}\left(x_{j, t}\right)^{2}+2 a_{2} x_{i, t-1} x_{j, t}+2 a_{3} x_{i, t-1}+2 a_{4} x_{j, t}+a_{5} \\
\equiv y_{t+1}^{\prime} Q_{i, k} y_{t+1}
\end{gathered}
$$

for $i=0$ (if $t$ is odd) or $i=1$ (if $t$ is even), where $\delta$ is strictly negative, and $k=t$ $\bmod 2$. Sub-section 3.2 will explain why the term $\delta\left(x_{i, t}\right)^{2}$ is included in (6) and why this inclusion does not affect the final solution (in terms of the choice variables of the original game).

Combining (3), (5) and (6), the four payoff matrices $Q_{i, k}(i=0,1 ; k=0,1)$ for the 
above game are given by:

$$
\begin{aligned}
Q_{0,0} & =\left[\begin{array}{ccccc}
a_{0} & 0 & 0 & a_{2} & a_{3} \\
0 & 0 & 0 & 0 & 0 \\
0 & 0 & 0 & 0 & 0 \\
a_{2} & 0 & 0 & a_{1} & a_{4} \\
a_{3} & 0 & 0 & a_{4} & a_{5}
\end{array}\right], \\
Q_{1,0} & {\left[\begin{array}{lllll}
a_{1} & 0 & 0 & a_{2} & a_{4} \\
0 & \delta & 0 & 0 & 0 \\
0 & 0 & 0 & 0 & 0 \\
a_{2} & 0 & 0 & a_{0} & a_{3} \\
a_{4} & 0 & 0 & a_{3} & a_{5}
\end{array}\right], } \\
Q_{0,1}= & {\left[\begin{array}{lllll}
\delta & 0 & 0 & 0 & 0 \\
0 & a_{1} & a_{2} & 0 & a_{4} \\
0 & a_{2} & a_{0} & 0 & a_{3} \\
0 & 0 & 0 & 0 & 0 \\
0 & a_{4} & a_{3} & 0 & a_{5}
\end{array}\right], }
\end{aligned}
$$

and

$$
Q_{1,1}=\left[\begin{array}{ccccc}
0 & 0 & 0 & 0 & 0 \\
0 & a_{0} & a_{2} & 0 & a_{3} \\
0 & a_{2} & a_{1} & 0 & a_{4} \\
0 & 0 & 0 & 0 & 0 \\
0 & a_{3} & a_{4} & 0 & a_{5}
\end{array}\right]
$$

The analysis of this section shows that the above two-symmetric-player linearquadratic alternating-move game can be reformulated as a simultaneous-move dynamic game with alternating payoff functions, provided that appropriate care is taken during the reformulation process. This simple example is helpful in understanding the 
approach used in the next section, in which a general multi-player linear-quadratic alternating-move game is considered.

\section{SOLUTION OF MULTI-PLAYER LINEAR-QUADRATIC ALTERNATING-MOVE GAMES}

This section considers a multi-player linear-quadratic alternating-move game (with the number of players, $n$, equal to or greater than 2) from period 0 to period $r-1$ (where $r$ is assumed to be a multiple of $n$ ) in which:

1. player $i(i=0,1, \ldots, n-1)$ chooses his action $z_{i, n \lambda+i}$ in period $n \lambda+i$ for some non-negative integer $\lambda$, and the control remains unchanged for $n$ periods (see Figure 1), ${ }^{1}$

2. the intertemporal payoff of each player is given by the expected present discounted value of a stream of single-period payoffs, which are quadratic functions of the choices of the players, and

3. the law of motion is linear (and, possibly, stochastic).

This game is called AM (which stands for "alternating moves"). Since it is more convenient to represent the payoff functions and the law of motion in terms of the variables of the reformulated game, they will be given later in Sub-section 3.2.

\footnotetext{
${ }^{1}$ As in Section 2, $z$ refers to choice variables of the alternating-move game, and $x$ refers to choice variables of the simultaneous-move game with alternating payoff functions. Moreover, to economize on the use of notation, some of the symbols are the same as those in Section 2, even though they refer to different games.
} 


\subsection{A linear-quadratic simultaneous-move dynamic game with alternating payoff functions}

Though the ultimate objective of this paper is to solve a multi-player linearquadratic alternating-move game, it is helpful to first consider a more general class of games: multi-player linear-quadratic simultaneous-move dynamic games with alternating payoff functions. Therefore, this sub-section introduces this class of games and defines relevant terms.

Consider a finite-horizon $n$-player $(n \geq 2)$ linear-quadratic dynamic game from period 0 to period $r-1$ (where $r$ is a multiple of $n$ ) in which: (a) all players (labelled as players 0 to $n-1$ ) move simultaneously at every period to maximize the expected present discounted value of a stream of single-period payoffs, (b) each player's singleperiod payoff functions are quadratic and alternating among $n$ functional forms, and (c) the law of motion is linear.

Let $x_{t}, y_{t}$ and $\theta_{t}$ be vectors of dimension $n, w$ and $v$ respectively. At any period $t$, each player is assumed to control just one variable $x_{i, t}$, and vector $x_{t} \equiv$ $\left(x_{0, t}, x_{1, t}, \ldots, x_{n-1, t}\right)^{\prime}$ represents the players' controls. Vector $y_{t}$ describes the state of the system at the beginning of period $t$, which may include a constant or the players' choice variables in previous periods. Vector $\theta_{t}$ represents the random variables, which are assumed to be realized after the players make their decisions at that period. The vector of random variables is also assumed to be serially uncorrelated with mean zero and finite covariance matrix $\Omega$, i.e., $E\left(\theta_{t} \theta_{t}^{\prime}\right)=\Omega$ for $t=0,1, \ldots, r-1$ where $E$ is the expectation operator. Therefore, the linear law of motion of the system is given by:

$$
y_{t+1}=f\left(y_{t}, x_{t}, \theta_{t}\right)=A y_{t}+B x_{t}+C \theta_{t}
$$

where $t=0,1, \ldots, r-1$, and the dimensions of matrices $A, B$ and $C$ can be deduced easily. 
Define the following $n$ functions, $f_{i}($.$) , for notational convenience:$

$$
f\left(y_{t}, x_{t}, \theta_{t}\right)=f_{i}\left(y_{t}, x_{i, t}, x_{-i, t}, \theta_{t}\right)
$$

where $i=0,1, \ldots, n-1, t=0,1, \ldots, r-1$, and $x_{-i, t} \equiv\left(x_{0, t}, \ldots, x_{i-1, t}, x_{i+1, t}, \ldots, x_{n-1, t}\right)^{\prime}$ is the $(n-1) \times 1$ vector of controls excluding $x_{i, t}$.

The intertemporal objective function of player $i(i=0,1, \ldots, n-1)$ is given by:

$$
E\left[\sum_{t=0}^{r-1}\left(\beta_{i}\right)^{t} U_{i, t}\right]
$$

where $\beta_{i}\left(0<\beta_{i}<1\right)$ is player $i$ 's discount factor and $U_{i, t}$ is the single-period payoff of player $i$ at $t$, which is represented by:

$$
U_{i, t}=U_{i, k}\left(y_{t}, x_{i, t}, x_{-i, t}, \theta_{t}\right)
$$

where $k$ is a non-negative integer smaller than or equal to $(n-1)$ such that

$$
k=t \bmod n,
$$

which means that there exists a non-negative integer $\lambda$ such that $t=n \lambda+k$ and $n \lambda \leq$ $t<n(\lambda+1)$. The above specification captures the fact that player $i$ 's single-period payoff functions are alternating among $n$ functional forms $U_{i, k}($.$) . Furthermore, it is$ assumed that $U_{i, t}$ quadratic and represented by:

$$
U_{i, t}=y_{t+1}^{\prime} P_{i, k} y_{t+1}=\left(A y_{t}+\sum_{j=0}^{n-1} b_{j} x_{j, t}+C \theta_{t}\right)^{\prime} P_{i, k}\left(A y_{t}+\sum_{j=0}^{n-1} b_{j} x_{j, t}+C \theta_{t}\right),
$$

where $b_{j}(w \times 1)$ is column $j$ of matrix $B$ in $(11)$, and $P_{i, k}$ is a symmetric $w \times w$ matrix.

The structure of the game and the definition of the value functions (to be discussed later) are summarized diagrammatically in Figure 2. 


\subsection{Reformulation of the original alternating-move game as a simultaneous- move dynamic game with alternating payoff functions}

There is a difference in structure between a simultaneous-move dynamic game with alternating payoff functions and an alternating-move game. To apply the framework in Sub-section 3.1 to game AM, a corresponding simultaneous-move dynamic game with alternating single-period payoff functions is constructed by:

1. Defining $x_{i, t}=z_{i, t}$ for all $t \bmod n=i$ (i.e. the periods when player $i$ moves),

2. Introducing dummy control variables $x_{i, t}$ for all $t$ and all $i$ such that $t \bmod$ $n \neq i$,

3. Representing the linear law of motion according to (11) after appropriately defining vectors $x_{t}, y_{t}$ and $\theta_{t}{ }^{2}$ and

4. Expressing the quadratic single-period payoff functions of the players in terms of the state variables $y_{t}$, according to (16).

An important point to note is that the dummy control variables do not appear in any player's payoff function in the original game AM. Therefore, there are multiple equilibria (with respect to these dummy control variables) in the above simultaneousmove dynamic game. In order to avoid this feature, a slightly different simultaneousmove game, to be called SMAP (which stands for "simultaneous moves, alternating payoffs"), is constructed by modifying the payoff functions of all players such that the term $\delta\left(x_{i, t}\right)^{2}$ (where $\left.\delta<0\right)$ is added to $U_{i, t}$ for all $t \bmod n \neq i .^{3}$ As a result, the

\footnotetext{
${ }^{2}$ To make the notation of an alternating-move game consistent with that in Sub-section 3.1, it is easily seen that the vector of controls $x_{t}$ in (11) includes not only the actual control variables (of the original game AM) but also the dummy control variables. See also the motivating example in Section 2 for the construction of control variables $x_{t}$ and state variables $y_{t}$.

${ }^{3}$ Technically, this step is introduced to prevent the non-invertibility of the matrix $H_{t} B$ in $(21)$. Non-invertibility of this matrix means that the game has either no equilibrium or multiple equilibria.
} 
single-period payoff function of player $i$ at period $t$ for the constructed game SMAP can be written as:

$$
U_{i, t}=y_{t+1}^{\prime} Q_{i, k} y_{t+1},
$$

where $Q_{i, k}$ (which is also symmetric) and $P_{i, k}$ in (16) are related by:

$$
Q_{i, k}=P_{i, k}
$$

for $k=i$, and

$$
y_{t+1}^{\prime} Q_{i, k} y_{t+1}=y_{t+1}^{\prime} P_{i, k} y_{t+1}+\delta\left(x_{i, t}\right)^{2}
$$

for $k \neq i$.

The relationships in (17), (18) and (19) capture the fact that in the constructed game SMAP, the single-period payoff function of a player is the same as that in game AM during the periods when he moves, but includes the dummy control variable in any other period. It will be seen in the proof of Proposition 1 that the optimal choices of the dummy control variables equal to zero, and so the solution (in terms of the control variables of the original game AM, and the maximized payoffs of the players) of the constructed game SMAP will be the same as that of the original game AM, after ignoring the dummy control variables.

\subsection{Solution of the game}

Each player is assumed to choose a Markov (or feedback) strategy, i.e., the control variable is a function of the state variables only. Player $i$ chooses his sequence of Markov strategies $\left\{x_{i, t}\left(y_{t}\right) ; t=0,1, \ldots, r-1\right\}$ to maximize the intertemporal payoff function (13) subject to the law of motion (11), the decision rules of other players, and the initial condition $y_{0}$.

The solution concept used in this paper is Markov perfect equilibrium (MPE). ${ }^{4} \mathrm{~A}$

\footnotetext{
${ }^{4}$ The terminology follows Maskin and Tirole (1987). Kydland (1975) and Basar and Olsder (1999) use the term 'feedback Nash equilibrium' for the same concept.
} 
sequence of Markov strategy vectors $\left\{x_{t}\left(y_{t}\right) ; t=0,1, \ldots, r-1\right\}$ constitutes a MPE of this game if for each player $i$, the sequence $\left\{x_{i, t}\left(y_{t}\right) ; t=0,1, \ldots, r-1\right\}$ maximizes player $i$ 's intertemporal payoff function (13), taking the decision rules of the other players $\left\{x_{-i, t}\left(y_{t}\right) ; t=0,1, \ldots, r-1\right\}$ as given. It can easily be seen that the above simultaneous-move dynamic game with alternating single-period payoff functions is a generalization of the simultaneous-move dynamic game with time-invariant singleperiod payoff functions considered in Kydland (1975).

A natural question arises in multi-agent decision problems is the possibility of multiple equilibria. In this paper, the equilibrium of the dynamic game is unique, which is guaranteed for the linear-quadratic model under assumption [A1] in Proposition 1.

To facilitate the derivation of the (unique) equilibrium of the above game, define the value functions for the players. Let $V_{i, t}\left(y_{t}\right)$ be the maximum intertemporal payoff that can be attained by player $i$ for the subgame starting with initial state $y_{t}$ at period $t$ and proceeding to the end of the game, with the optimal Markov strategies of the other $(n-1)$ players taken as $\left\{x_{-i, s}\left(y_{s}\right) ; s=t, t+1, \ldots, r-1\right\}$. The above definition leads to the following recursive relationship of value functions for $t=0,1, \ldots, r-1$ :

$V_{i, t}\left(y_{t}\right)=\max _{q_{i, t}\left(y_{t}\right)} E\left\{U_{i, k}\left[y_{t}, q_{i, t}\left(y_{t}\right), x_{-i, t}\left(y_{t}\right), \theta_{t}\right]+\beta_{i} V_{i, t+1}\left[f_{i}\left(y_{t}, q_{i, t}\left(y_{t}\right), x_{-i, t}\left(y_{t}\right), \theta_{t}\right)\right]\right\}$

where $k$ is defined in (15). Without loss of generality, it is assumed that $V_{i, r}\left(y_{r}\right)=0$ for all possible values of $y_{r}$ at the end of the game.

For the constructed game SMAP with quadratic and alternating payoff functions, Proposition 1 gives the (computationally verifiable) conditions for existence and uniqueness of the MPE, and an algorithm to compute the equilibrium.

Proposition 1 Assume that [A1]:

$$
b_{i}^{\prime}\left(Q_{i, k}+\beta_{i} S_{i, t+1}\right) b_{i}<0
$$


for $i=0,1, \ldots, n-1$ and $t=0,1, \ldots, r-1$ where $k$ is defined in (15) and $S_{i, t+1}$ is defined recursively in (23). Then there is a unique MPE to the constructed linearquadratic simultaneous-move game SMAP. The equilibrium decision rules $x_{t}\left(y_{t}\right)$ and the value functions $V_{i, t}\left(y_{t}\right)$ can be computed recursively by:

$$
\begin{aligned}
& x_{t}\left(y_{t}\right)=-\left(H_{t} B\right)^{-1} H_{t} A y_{t}, \\
& V_{i, t}\left(y_{t}\right)=y_{t}^{\prime} S_{i, t} y_{t}+c_{i, t} \\
& S_{i, t}=A^{\prime}\left[I-B\left(H_{t} B\right)^{-1} H_{t}\right]^{\prime}\left(Q_{i, k}+\beta_{i} S_{i, t+1}\right)\left[I-B\left(H_{t} B\right)^{-1} H_{t}\right] A \text {, } \\
& c_{i, t}=\operatorname{trace}\left[C^{\prime}\left(Q_{i, k}+\beta_{i} S_{i, t+1}\right) C \Omega\right]+\beta_{i} c_{i, t+1}, \\
& H_{t}=\left[\begin{array}{c}
b_{0}^{\prime}\left(Q_{0, k}+\beta_{0} S_{0, t+1}\right) \\
b_{1}^{\prime}\left(Q_{1, k}+\beta_{1} S_{1, t+1}\right) \\
\cdot \\
\cdot \\
b_{n-1}^{\prime}\left(Q_{n-1, k}+\beta_{n-1} S_{n-1, t+1}\right)
\end{array}\right]
\end{aligned}
$$

where $S_{i, r}=0, c_{i, r}=0, I$ is an identity matrix of dimension $w$, and the trace of a square matrix is the sum of its diagonal elements.

The proof of Proposition 1, which is based on Basar and Olsder (1999) and especially Kydland (1975), is given in an Appendix available from the author upon request. Note that assumptions (i) and (ii) in Theorem 1 of Kydland (1975) represent respectively the second order condition and the invertibility of matrix $H_{t} B$. In the constructed game SMAP considered in this paper, it is observed from the proof that the invertibility of $H_{t} B$ in (21) is guaranteed automatically. As a result, only one assumption, [A1], is required for Proposition 1.

To summarize, the above analysis suggests the following algorithm to obtain the equilibrium of the multi-player linear-quadratic alternating-move game AM: 
1. Construct $Q_{i, k}$ of game SMAP from $P_{i, k}$ for all $i$ and $k$ according to (18) and (19).

2. Start with $S_{i, r}=0$ and $c_{i, r}=0$ for all $i$.

3. Calculate the equilibrium decision rules and value functions for $i=0,1, \ldots, n-1$ and $t=0,1, \ldots, r-1$ recursively according to (21) to (25). Note that the validity of assumption [A1] can be checked computationally for every player and every period.

4. The solution to the original alternating-move game AM can be obtained from that of the constructed game SMAP, after ignoring the equilibrium values of all dummy control variables.

\subsection{Limit of the finite-horizon games as the horizon grows}

An interesting follow-up step is to obtain the MPE of the corresponding infinitehorizon alternating-move game, interpreted as the limit of a sequence of finite-horizon games as the number of periods, $r$, tends to infinity. A nice property in considering the limiting model is that the equilibrium decision rules, if they exist, will be timeinvariant. $^{5}$ The computation algorithm described above is applicable, and one can obtain the limiting equilibrium decision rules and value functions as arbitrarily close

\footnotetext{
${ }^{5}$ In an infinite-horizon alternating-move game, the equilibrium decision rules and the value functions take $n$ alternating functional forms and do not depend on calendar time. For example, the value functions in (20) are replaced by $V_{i, t} \bmod n\left(y_{t}\right)$ and $V_{i,(t+1)} \bmod n\left(y_{t+1}\right)$ respectively. Following similar analysis as in Proposition 1, it can be shown that the equilibrium decision rules and the value functions can be calculated recursively by (21) to (25) with the subscript $t$ in $H_{t}, S_{i, t}, c_{i, t}$ and $V_{i, t}$ (.) being replaced $t \bmod n$, and subscript $t+1$ in $S_{i, t+1}$ and $c_{i, t+1}$ being replaced by $(t+1) \bmod$ $n$. Similar interpretation and results for the infinite-horizon simultaneous-move dynamic games are found in Kydland (1975).
} 
as one wants, provided that they converge. ${ }^{6}$

As an example, consider the infinite-horizon version of the two-symmetric-player linear-quadratic alternating-move games in Section 2 that satisfy assumption [A1] of Proposition 1, which can be verified computationally (at every step of the calculation). ${ }^{7}$ By iterating (23) with specific values of $\beta, a_{0}, a_{1}, a_{2}, a_{3}, a_{4}$ and $a_{5}$ substituted in $Q_{i, k}(i=0,1 ; k=0,1)$ according to $(7)$ to $(10)$, the matrices $S_{i, t}$ are found to converge numerically to two alternating matrices for each player. It can be shown that, when $t$ is even,

$$
\left[\begin{array}{l}
x_{0, t} \\
x_{1, t}
\end{array}\right]=\left[\begin{array}{lllll}
0 & \gamma & 0 & 0 & \alpha \\
0 & 0 & 0 & 0 & 0
\end{array}\right] y_{t}=\left[\begin{array}{c}
\alpha+\gamma x_{1, t-1} \\
0
\end{array}\right],
$$

and when $t$ is odd,

$$
\left[\begin{array}{l}
x_{0, t} \\
x_{1, t}
\end{array}\right]=\left[\begin{array}{lllll}
0 & 0 & 0 & 0 & 0 \\
\gamma & 0 & 0 & 0 & \alpha
\end{array}\right] y_{t}=\left[\begin{array}{c}
0 \\
\alpha+\gamma x_{0, t-1}
\end{array}\right],
$$

where $\alpha$ and $\gamma$ are coefficients depending on $\beta, a_{0}, a_{1}, a_{2}, a_{3}, a_{4}$ and $a_{5}$. As expected, all dummy control variables are zero at the equilibrium. In terms of the original alternating-move game, the equilibrium reaction functions are given by:

$$
z_{t \bmod 2, t}=\alpha+\gamma z_{(t-1)} \bmod 2, t-1 .
$$

\footnotetext{
${ }^{6}$ Maskin and Tirole (1987) use a contraction mapping argument to show that, as the horizon increases, the finite-horizon equilibrium strategies of their model (with a specific functional form) converge to their infinite-horizon counterparts. While similar proofs should ideally be performed for other problems before the suggested algorithm is applied, this line of investigation is not pursued in Kydland (1975) and this paper which focus on how the solution can be represented in a simple recursive form and therefore can be obtained computationally. See Kydland (1975, section 5) and Maskin and Tirole (1987, section 4) for more discussions about the existence and convergence issues for the infinite-horizon games.

${ }^{7} \mathrm{~A}$ necessary condition for this assumption to hold is $b_{i}^{\prime} Q_{i, k} b_{i}<0$. While this condition is not satisfied for every possible quadratic payoff function represented by (2), it is satisfied for many economic models such as Maskin and Tirole (1987) and De Fraja (1993).
} 
The above results are applicable to the class of two-symmetric-player linear-quadratic alternating-move games including Maskin and Tirole (1987), De Fraja (1993) and Cahuc and Kempf (1997). ${ }^{8}$

\section{A WAGE SETTING GAME WITH MORE THAN TWO PLAYERS}

Lau (1996) examines the aggregate pattern of wage adjustment in a two-sector model with strategic complementarity and negative externality, and shows that wage setters prefer moving alternatingly when they interact strategically in a decentralized environment. In that model, wage setters in a particular sector care about their real wages, but they can only control directly their nominal wages since the general price level is also affected by the decisions elsewhere in the economy. Moreover, it is assumed that an increase (resp. a decrease) in the nominal wage of one sector will, other things being equal, decrease (resp. increase) the payoffs of other agents, and that the optimal reaction to an increase (resp. a decrease) in nominal wages elsewhere in the economy is to have one's own nominal wage increased (resp. decreased).

This section examines whether the result that nonsynchronization is the preferred timing pattern in the above model is robust to the number of wage setters in the economy. ${ }^{9}$ Specifically, analysis using the solution method in Section 3 is carried out

\footnotetext{
${ }^{8}$ For example, the single-period payoff function (15) of Maskin and Tirole (1987) can be expressed in the form of (2) of this paper with $a_{0}=-1, a_{1}=-1, a_{3}=d / 2$ and $a_{2}=a_{4}=a_{5}=0$. It can be shown numerically that coefficients $\gamma$ and $\alpha$ of the equilibrium reaction functions (28) obtained by the solution algorithm suggested in Proposition 1 are the same as those obtained by (20) and (21) of Maskin and Tirole (1987).

${ }^{9}$ Note that Fethke and Policano (1986) and this paper only consider whether staggered wage adjustment is Pareto optimal or not in an economy with many sectors, but not the more difficult question of whether and how the optimal timing pattern will arise as the equilibrium outcome. It is quite difficult in general to derive the aggregate timing pattern endogenously in a multi-sector model (say, by allowing the players the choice in moving from one cohort to another by waiting an extra
} 
for a three-sector model, and then the implications of the results of this model for an economy with more sectors is discussed.

In an economy with $n(n \geq 2)$ sectors, the intertemporal payoff function of each wage setter is assumed to be given by (13) with $\beta_{i}=\beta$, the horizon $r$ tends to infinity, and

$$
U_{i, t}=\left(z_{i, t}-p_{t}\right)\left[h-\left(z_{i, t}-p_{t}\right)\right]-g\left(m_{t}-p_{t}\right)^{2}
$$

where $g(0<g<1)$ and $h(h>0)$ are constants, $z_{i, t}$ is nominal wage (in log) of sector $i$ at $t, p_{t}$ is the general price level (in log) at $t$ and is given by:

$$
p_{t}=\frac{1}{n} \sum_{j=0}^{n-1} z_{i, t}+e_{t},
$$

and $m_{t}$ and $e_{t}$ are money and price shocks at $t$. The money and price shocks are assumed to be uncorrelated zero-mean random processes and are realized at the beginning of a period. ${ }^{10}$ Note that the above model is of infinite-horizon and is a generalization of the two-sector model in Lau (1996); a detailed discussion (for the two-sector version) of the model can be found in that paper.

When the number of sectors increases beyond two, however, there is no single measure of the degree of synchronization. To highlight the role of strategic behavior in affecting the aggregate pattern of wage negotiation, this section compares the two polar cases: complete synchronization versus uniform staggering.

period, as specified in Maskin and Tirole, 1987). Maskin and Tirole (1987, p. 963), Ball and Romer (1989, p. 186) and Lau (1996, p. 1651) discuss some issues related to the modelling of equilibrium timing pattern and discuss why the Pareto optimal timing pattern is likely to be the equilibrium outcome.

${ }^{10}$ In order to apply the solution method suggested in this paper, vector $\theta_{t}$ (which is assumed to be realized after the players make their decisions at period $t)$ is defined as $\theta_{t}=\left(m_{t+1}, e_{t+1}\right)^{\prime}$. 


\subsection{Complete synchronization}

Under this timing pattern, all wage contracts last for $n$ periods and all $n$ sectors determine wages at the same time, say, when $t$ is a multiple of $n$. Taking the nominal wages of the other $(n-1)$ sectors as given, wage setter $i$ chooses $z_{i, n \lambda}$ to maximize

$$
E\left[\sum_{t=n \lambda}^{n(\lambda+1)-1} \beta^{(t-n \lambda)} U_{i, t}\right],
$$

where $U_{i, t}$ is given by (29). Because of symmetry, it can easily be shown that the equilibrium wage is given by:

$$
z_{i, n \lambda}^{S Y N C}=\frac{\left(1-\beta^{n}\right)(n-1) h+2(1-\beta) g m_{n \lambda}+2(1-\beta)(n-1-g) e_{n \lambda}}{2\left(1-\beta^{n}\right) g} .
$$

\subsection{Uniform staggering}

Under this pattern, the wage contracts of the $n$ sectors are renewed alternatingly such that wage setter $i$ makes decision at period $n \lambda+i$. Each contract lasts for $n$ periods and the contract wage is assumed to be constant throughout the duration.

The reformulation of this alternating-move game to the framework of Sub-section 3.2 is similar to that used in the example of Section 2. Applying the suggested algorithm to the three-sector model, the matrices $S_{i, t}$ defined in (23) are found to converge numerically to three alternating matrices for each player. As a result, the equilibrium reaction functions for the three-sector wage setting game is of the following form:

$$
z_{t \bmod 3, t}=\phi_{0}+\phi_{1} z_{(t-1) \bmod 3, t-1}+\phi_{2} z_{(t-2) \bmod 3, t-2}+\phi_{3} m_{t}+\phi_{4} e_{t}
$$

where $\phi_{0}, \phi_{1}, \phi_{2}, \phi_{3}$ and $\phi_{4}$ are coefficients depending on $\beta$ and $g$. Some computed values are shown in Table 1. 


\subsection{A comparison of synchronized and staggered wage adjustment}

In the two-sector version of the wage setting game, the results in Lau (1996, p. 1652) suggest that there are two factors influencing the merits of staggered adjustment as compared to synchronization: the importance of strategic dependence and that of the random shocks. Specifically, the steady state payoff of each of the players under staggered wage adjustment is always higher than its counterpart under synchronization if there is no random shock; moreover, the difference of the steady state payoffs under staggered and synchronized wage adjustment is increasing in the variance of the price shock but is decreasing in the variance of the money shock.

Regarding the benefit provided by staggered wage adjustment under strategic and dynamic interaction, the intuition is as follows. By choosing wages at different times, the action of each wage setter will not be offset by that of his opponent. Therefore, a player does not need to set a very high nominal wage. Because of the presence of strategic complementarity, this choice of a low nominal wage further induces his opponent to follow in the future. As a result, both players obtain higher payoffs because of the presence of negative externality (with the lower nominal wages leading to higher aggregate demand).

As the main concern of this section is whether the strategic benefit of a staggered timing pattern is robust with respect to the number of wage setters, the effect of the random shocks is of secondary importance and thus not examined. In the following analysis, the deterministic components of the players' steady state payoffs under these two regimes are compared. Under complete synchronization, the deterministic component of the steady state payoff of each of the three wage setters is given by $-g\left(z^{S Y N C}\right)^{2}$ where $z^{S Y N C}=h / g$; see (32). Under uniform staggering, this is given by $-g\left(z^{S T A G}\right)^{2}$ where $z^{S T A G}=\phi_{0} h /\left(1-\phi_{1}-\phi_{2}\right)$, as can be deduced from (33). Table 2 shows the difference of the deterministic components of the steady state 
payoffs under these two regimes for some parameter pairs $(\beta, g)$ in two- and threesector models respectively. ${ }^{11}$

From Table 2, it can be seen that all terms are positive, implying that uniform staggering is preferred to complete synchronization for all parameter combinations of both the two- and three-sector models. A more important observation is that for a particular $(\beta, g)$ combination, the difference is increasing in the number of sectors in the economy. (Extensive computational checks suggest that this is true for all parameter combinations of $0<\beta<1$ and $0<g<1$.) These results suggest that a staggered timing pattern will still be preferred in a decentralized economy with many wage setters when they interact strategically.

What are the intuitions of the above results? The fundamental source of conflict in this wage setting model is the interdependence and strategic interactions among the wage setters. When they move simultaneously, they tend to set a 'too-high' level of nominal wage due to the usual strategic consideration. Nevertheless, each wage setter's action is offset by his opponent at the equilibrium, with the resulting high nominal wages in all sectors leading to a high aggregate price level. Consequently, all wage setters suffer due to the low aggregate demand. When the number of wage setters increases (and therefore nominal wage in each sector has less influence on the aggregate price level, as can be observed in (30)), the externality problem of a higher nominal wage in any sector on the welfare of other wage setters (through the price level) becomes more severe. As the strategic benefit of staggered wage adjustment lies in its ability in overcoming the externalities involved in decentralized wage setting, ${ }^{12}$ it is logical to conjecture that such benefit of a staggered timing pattern becomes

\footnotetext{
${ }^{11}$ For the two-sector model, the corresponding number is calculated from the deterministic component of (7) in Lau (1996). Alternatively, it can be obtained by the algorithm suggested in Proposition 1.

${ }^{12}$ See more discussions about issues associated with externalities in wage adjustment in Layard et al. (1991).
} 
more important when the number of wage setters increases. The comparisons of the wage setters' payoffs under the two regimes in Table 2 confirm this conjecture.

\section{CONCLUSION}

Following the analysis of Cyert and DeGroot (1970) and Maskin and Tirole (1987), linear-quadratic alternating-move games with two players have recently been applied in many papers. Perhaps because of the complexity in analyzing an alternating-move game with three players or more, only two-player games have been considered. In order to study an alternating-move dynamic game with more than two players, this paper develops a solution method by making use of the similarity of this game and a simultaneous-move dynamic game with alternating payoff functions. The solution procedure is applicable to the class of multi-player linear-quadratic alternating-move games, including the cases of (a) more than two players, and (b) asymmetric intertemporal payoff functions of the players (in terms of either different discount factors or different single-period payoff functions). ${ }^{13}$

The solution method discussed in this paper is developed for an alternating-move dynamic game in which only one player moves at each time period. This specification is a natural extension of the two-player alternating-move game widely used in the literature. The proposed solution procedure can easily be extended to other cases in which the number of periods that the control remains unchanged is different from the number of players. However, in such an alternating-move game in which two or more players move at a particular period, an assumption which guarantees the invertibility of a matrix similar to $H_{t} B$ in (21) is also needed; see assumption (ii) in Theorem 1

\footnotetext{
${ }^{13}$ As an example, the asymmetric single-period payoff function (1) of Tanaka (1994) can be expressed in a form similar to that of the four matrices $Q_{i, k}(i=0,1 ; k=0,1)$ of Equations (7) to (10) of this paper with parameters $a_{0}=-1, a_{1}=-1, a_{3}^{i}=\left(d+s_{i}\right) / 2$ and $a_{2}=a_{4}=a_{5}=0$, where $a_{3}$ in $Q_{i, 0}$ and $Q_{i, 1}(i=0,1)$ are replaced by $a_{3}^{i}$.
} 
of Kydland (1975) as well. This modification is rather straightforward; moreover, the validity of this assumption can also be checked computationally.

Applying the proposed algorithm in Proposition 1 to study the timing pattern of wage adjustment in a three-sector model, it is found that the deterministic component of the steady state payoff of each of the three wage setters under uniform staggering is higher than its counterpart under complete synchronization. The result supports the conjecture that the benefit provided by staggered wage adjustment is robust to the number of wage setters when they interact strategically.

\section{REFERENCES}

[1] Ball, L. and D. Romer (1989), "The Equilibrium and Optimal Timing of Price Changes," Review of Economic Studies, Vol. 56, 179-198.

[2] Basar, T. and G. J. Olsder (1999), Dynamic Noncooperative Game Theory, Second Edition, Philadelphia: SIAM.

[3] Cahuc, P. and H. Kempf (1997), "Employment and Wage Bargaining in an Open Monetary Union", Review of International Economics Vol. 5, Supplement, S92S110.

[4] Cyert, R. M. and M. H. DeGroot (1970), "Multiperiod Decision Models with Alternating Choice as the Solution to the Duopoly Problem," Quarterly Journal of Economics Vol. 84, 410-429.

[5] De Fraja, G. (1993), "Staggered vs. Synchronised Wage Setting in Oligopoly," European Economic Review Vol. 37, 1507-1522.

[6] Fethke, G. and A. Policano (1984), "Wage Contingencies, the Pattern of Negotiation and Aggregate Implications of Alternative Contract Structures," Journal of Monetary Economics, Vol. 14, 151-170. 
[7] Fethke, G. and A. Policano (1986), "Will Wage Setters Ever Stagger Decisions?" Quarterly Journal of Economics, Vol. 101, 867-877.

[8] Fischer, S. (1977), "Long Term Contracts, Rational Expectations, and the Optimal Money Supply Rule," Journal of Political Economy, Vol. 85, 163-190.

[9] Kydland, F. (1975), "Noncooperative and Dominant Player Solutions in Discrete Dynamic Games," International Economic Review, Vol. 16, 321-335.

[10] Lau, S.-H. P. (1996), "Aggregate Pattern of Time-dependent Adjustment Rules, I: A Game-theoretic Analysis of Staggered versus Synchronised Wage Setting," Economic Journal, Vol. 106, 1645-1658.

[11] Layard, R., S. Nickell and R. Jackman (1991), Unemployment - Macroeconomic Performance and the Labour Market, Oxford University Press.

[12] Maskin, E. and J. Tirole (1987), "A Theory of Dynamic Oligopoly, III: Cournot Competition," European Economic Review, Vol. 31, 947-968.

[13] Tanaka Y. (1994), "Export Subsidies Under Dynamic Duopoly," European Economic Review, Vol. 38, 1139-1151.

[14] Taylor, J. B. (1980), "Aggregate Dynamics and Staggered Contracts," Journal of Political Economy, Vol. 88, 1-23. 
Table 1: Equilibrium reaction functions for the three-sector alternating-move wage game

\begin{tabular}{|c|c|c|c|c|c|}
\hline$\beta \backslash g$ & 0.1 & 0.3 & 0.5 & 0.7 & 0.9 \\
\hline \multirow[t]{5}{*}{0.1} & 0.77 & 0.72 & 0.68 & 0.65 & 0.62 \\
\hline & 0.48 & 0.40 & 0.33 & 0.27 & 0.22 \\
\hline & 0.44 & 0.37 & 0.31 & 0.25 & 0.20 \\
\hline & 0.069 & 0.19 & 0.31 & 0.41 & 0.50 \\
\hline & 1.31 & 1.10 & 0.92 & 0.76 & 0.61 \\
\hline \multirow[t]{5}{*}{0.3} & 0.85 & 0.77 & 0.71 & 0.66 & 0.62 \\
\hline & 0.50 & 0.40 & 0.32 & 0.26 & 0.20 \\
\hline & 0.38 & 0.31 & 0.25 & 0.21 & 0.17 \\
\hline & 0.060 & 0.16 & 0.25 & 0.33 & 0.41 \\
\hline & 1.15 & 0.93 & 0.76 & 0.62 & 0.50 \\
\hline \multirow[t]{5}{*}{0.5} & 0.93 & 0.79 & 0.72 & 0.66 & 0.62 \\
\hline & 0.50 & 0.38 & 0.30 & 0.24 & 0.18 \\
\hline & 0.33 & 0.26 & 0.21 & 0.17 & 0.13 \\
\hline & 0.053 & 0.14 & 0.21 & 0.27 & 0.33 \\
\hline & 1.00 & 0.78 & 0.62 & 0.50 & 0.40 \\
\hline \multirow[t]{5}{*}{0.7} & 0.98 & 0.80 & 0.71 & 0.66 & 0.62 \\
\hline & 0.48 & 0.35 & 0.27 & 0.21 & 0.17 \\
\hline & 0.28 & 0.21 & 0.17 & 0.13 & 0.11 \\
\hline & 0.045 & 0.11 & 0.17 & 0.22 & 0.26 \\
\hline & 0.85 & 0.64 & 0.51 & 0.41 & 0.32 \\
\hline \multirow[t]{5}{*}{0.9} & 0.99 & 0.79 & 0.70 & 0.65 & 0.61 \\
\hline & 0.45 & 0.32 & 0.25 & 0.19 & 0.15 \\
\hline & 0.23 & 0.17 & 0.14 & 0.11 & 0.086 \\
\hline & 0.037 & 0.092 & 0.14 & 0.18 & 0.21 \\
\hline & 0.70 & 0.52 & 0.41 & 0.33 & 0.26 \\
\hline
\end{tabular}

Note:

For each ( $\beta$, g) combination, the five numbers represent respectively the coefficients $\phi_{0}, \phi_{1}, \phi_{2}, \phi_{3}$ and $\phi_{4}$ of the equilibrium reaction function in (33) of the main text. 
Table 2: The difference between a wage setter's payoffs under staggering and synchronization

\begin{tabular}{c|ccccc}
$\beta \backslash \mathrm{g}$ & 0.1 & 0.3 & 0.5 & 0.7 & 0.9 \\
\hline \multirow{2}{*}{0.1} & 0.68 & 0.15 & 0.058 & 0.022 & 0.005 \\
& 1.50 & 0.42 & 0.21 & 0.12 & 0.078 \\
0.3 & 1.47 & 0.34 & 0.13 & 0.054 & 0.013 \\
& 4.53 & 1.25 & 0.63 & 0.37 & 0.23 \\
0.5 & 1.86 & 0.45 & 0.18 & 0.075 & 0.019 \\
& 6.84 & 1.90 & 0.96 & 0.57 & 0.36 \\
0.7 & 2.09 & 0.52 & 0.22 & 0.091 & 0.023 \\
& 8.27 & 2.33 & 1.19 & 0.72 & 0.46 \\
0.9 & 2.21 & 0.57 & 0.24 & 0.10 & 0.026 \\
& 9.05 & 2.61 & 1.35 & 0.82 & 0.54
\end{tabular}

Note:

For each ( $\beta, g)$ combination, the first (resp. second) number represents the deterministic component of

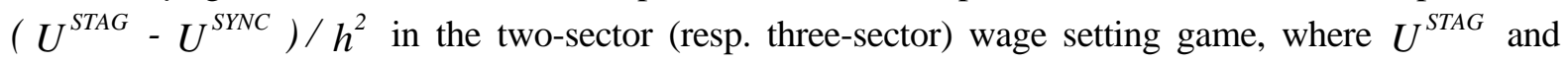
$U^{S Y N C}$ are respectively the steady state payoffs of a player under uniform staggering and complete synchronization. 
Figure 1: A multi-player alternating-move dynamic game

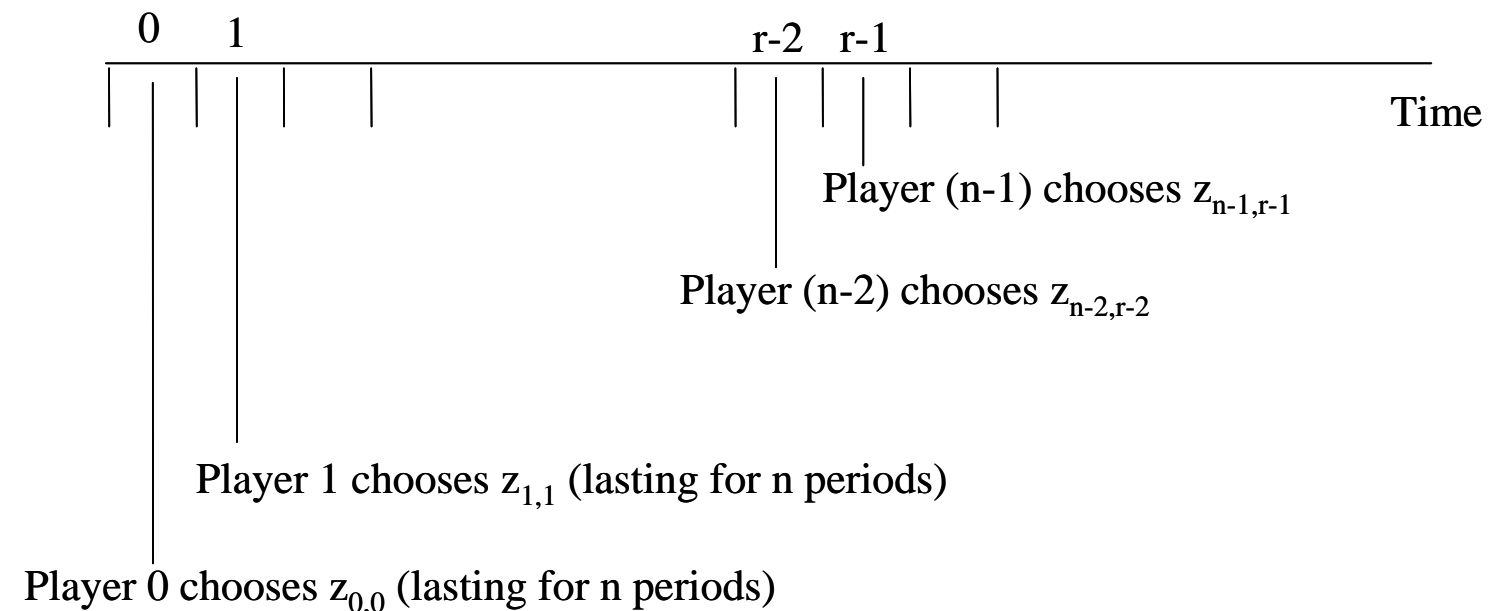

Note: $r$ is a multiple of $n$.

Figure 2: A multi-player simultaneous-move dynamic game with alternating payoff functions

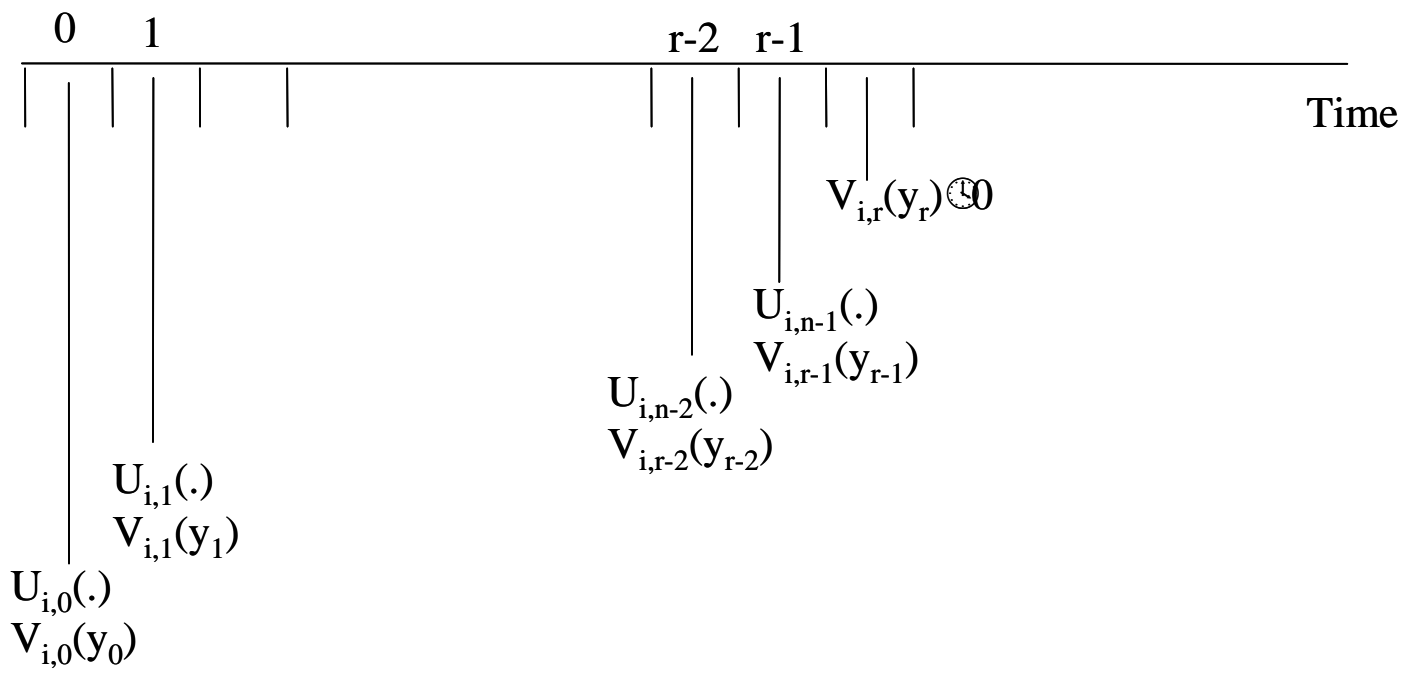

Note: $r$ is a multiple of $n$. 